\title{
Routines of resistance: an ethnography of the care of people living with dementia in acute hospital wards and its consequences
}

Katie Featherstone ${ }^{1}$, Andy Northcott ${ }^{2}$, Jackie Bridges ${ }^{3}$

${ }^{1}$ Cardiff University, School of Healthcare Sciences, Cardiff, UK. @drkfeatherstone ${ }^{2}$ De Montfort University, School of Allied Health Sciences, Leicester, UK @andynorthcott

${ }^{3}$ Faculty of Health Sciences, University of Southampton, Southampton, UK

\section{Corresponding Author}

Katie Featherstone.

Reader in Sociology and Medicine

Cardiff University, School of Healthcare Sciences, 13-19 Eastgate House, 43-35

Newport Road Cardiff, CF24 0AB, UK

featherstonek@cardiff.ac.uk

02920687689

\section{Abstract \\ Background}

There is little research examining resistance, refusal or rejection of care by people living with dementia within acute hospital wards despite the prevalence of dementia in adult hospital populations.

\section{Objectives}

To explore the ways in which resistance to care manifests within the acute setting and is understood, classified and subsequently managed by ward staff.

\section{Design}

Ethnography

\section{Setting}

Acute medical units and trauma and orthopaedic wards in five NHS hospitals in England and Wales.

\section{Participants}

People living with dementia and nursing team members (registered nurses and health care assistants) on participating wards. 


\section{Methods}

Observational fieldwork and ethnographic interviews collected over a period of 20 months (155 days of non-participant observation (minimum 2 hours, maximum 12 hours, total hours: 680) focusing on staff delivering care to patients with dementia. Interviewees included patients, visitors, and staff working on and visiting the ward. Data collection and analysis drew on the theoretical sampling and constant comparison techniques of grounded theory. 


\section{Results}

We found that resistance to care by people living with dementia was a routine and expected part of everyday care in the participating acute hospital settings. The timetabled rounds of the ward (mealtimes, medication rounds, planned personal care) significantly shaped patient and staff experiences and behaviours. These routinized ward cultures typically triggered further patient resistance to bedside care. Institutional timetables, and the high value placed on achieving efficiency and reducing perceived risks to patients, dictated staff priorities, ensuring a focus on the delivery of essential everyday planned care over individual patient need or mood in that moment. Staff were thus trapped into delivering routines of care that triggered patterns of resistance.

\section{Conclusions}

Nursing staff struggle to respond to the needs of people living with dementia in acute care settings where the institutional drivers of routines, efficiency and risk reduction are not mediated by clinical leadership within the ward. Cycles of resistance in response to organisationally mandated timetables of care can result in poor care experiences for patients, and emotional and physical burnout for staff. More research is needed into how institutional goals can be better aligned to recognise the needs of a key hospital population: people living with dementia.

\section{Keywords}

Dementia

Ethnography

Hospitals

Medication compliance

Nursing staff, Hospital

Patient autonomy

Patient compliance

Professional-Patient Relations

Treatment refusal

\section{Funding Acknowledgement:}

This project was funded by the National Institute for Health Research Health Services and Delivery Research programme (project number 13/10/80) 


\section{Department of Health and Social Care Disclaimer:}

The views and opinions expressed therein are those of the authors and do not necessarily reflect those of the Health Services and Delivery Research programme, NIHR, NHS or the Department of Health and Social Care.

\section{Contribution of the Paper}

\section{What is known about this topic:}

- People living with dementia are one of the largest patient groups admitted to acute hospital wards in the UK and are known to have poor outcomes.

- Resistance and refusal of care by people living with dementia is a commonly reported feature of caring work in institutional and home settings, particularly around nutritional intake and medication.

- Few have explored the phenomenon in acute hospital wards, nor is there evidence for how it should be managed by professionals caring for people living with dementia within these settings.

\section{What this paper adds:}

- This paper provides in-depth ethnographic evidence identifying high levels of resistance during timetabled care; every patient living with dementia observed, resisted care to some extent during observations within acute hospital wards across England and Wales.

- Identifies that ward staff felt constrained into delivering routines of care that involved short term management and containment practices, which in turn, typically triggered longer-term entrenched patterns of resistance and escalation.

- Identifies that institutional timetables, and the high value set on efficiency and reducing perceived risks, dictated ward priorities, led to a focus on the organisation and delivery of scheduled care over patient need. 


\section{Routines of resistance: an ethnography of the care of people living with dementia in acute hospital wards and its consequences}

\section{Introduction}

Resistance, refusal or rejection of care by people living with dementia is a commonly reported feature of caring work within institutional and home settings (Kable et al, 2012). Most research examining this phenomenon has taken place in long-term care settings (Ishii et al, 2012), but few have examined the phenomenon in acute settings (Clissett et al, 2013; Dewing \& Dijk 2014; Haughton et al, 2016), despite a dementia diagnosis becoming a growing feature of adult hospital populations internationally (Prince et al, 2016).

Resistance towards everyday care by people living with dementia is commonly associated with an admission to an acute care hospital (Moyle et al, 2008; Dewing \& Dijk, 2014.). It can include resistance and refusal towards everyday necessities of a hospital admission including food, hydration, medication, personal care and toileting. While resistance is not consistently defined in the literature (Ishii et al, 2012), at its core these behaviours are usually characterised as non-compliant behaviour in response to healthcare staff (Kable et al, 2012). Resistance is commonly perceived by clinical staff as a deviant behaviour, resulting in people living with dementia becoming labelled as a 'difficult' patient group (Moyle et al, 2008).

Arguably, the acute care environment itself triggers resistance amongst this patient group. Reviews suggest the built environment underpins patient responses to care (Moyle et al, 2008; Dewing \& Dijk, 2014). The round the clock backdrop of bright lights, alarms, voices, and human traffic make the acute hospital setting both threatening and frustrating for people living with dementia, but these findings are not definitive. Responses to such stimuli within acute wards may fall within the range of behaviours loosely conceptualised as resistance of care (Pizzacalla et al, 2015; Ishii et al. 2012). In addition, key staff responses to resistance of care often include restraint, sedation (Pizzacalla et al, 2015) or deprivation of liberty (Sangars et al, 2014) can become embedded (albeit undesirable) features of hospital cultures. However, the hospital social environment and its relationship to patient resistance is underexamined (Keady \& Jones, 2010; Rantala et al, 2014; Pizzacalla et al, 2015; Cheong et 
al, 2016). Overall, there is a lack of evidence documenting the extent of resistance to care, how it manifests, or how it should be managed, within the acute hospital setting (Werner et al, 2002; Dewing \& Dijk, 2014; Pizzacalla et al. 2015).

In this paper, the concept of resistance is emergent and 'in the making', surfacing in the ways people interpret and respond to each other during the mundane encounters, interactions and the everyday routine work of the ward. The actual act of resistance is always context bound and dependent on many factors. Thus, within this paper, rather than classifying behaviours as distinct entities, the authors use the term 'resistance' and show the ways in which these manifest as part of a continuum of responses people living with dementia have to the ways in which their care is delivered at the bedside and their wider experiences of their admission to an acute hospital ward. In turn, the ways in which staff are also trapped in this cycle of delivering fast-paced, timetabled care, and its consequences, are explored.

\section{Methods}

In order to explore the ways in which resistance to care manifests within the acute setting and is understood, classified, and subsequently managed by ward staff, we utilised an ethnographic approach. In the context of understanding how healthcare services within hospital settings are delivered and the organisation underlying its delivery, ethnography allows us to examine the everyday routine behaviours of individuals, both within and across multi- disciplinary teams (Quinlan, 2009) and the ways in which the social and institutional forces shape and influence the work of health care providers (Greenhalgh \& Swinglehurst, 2011). This methodological approach enabled us to understand how staff respond to care needs, the ways in which they account for and make sense of their responses, and also to follow the consequences of their actions and interactions.

This paper presents our analysis of ethnographic observations of staff and patient interactions before, during, and following, the care of people living with dementia and ethnographic interviews with staff during their work. A wide range of other data (ethnographic observation of a wide range of care work, ethnographic and indepth interviews with staff, and case studies with patients and families) was also collected and is reported elsewhere (Featherstone et al in press). 
Five hospitals were purposefully selected to represent a range of hospitals types: 2 large University teaching hospitals (B and D), 2 medium sized general hospitals (A and $C$ ) and 1 smaller general hospital (E)), geographical locations (urban (A), innercity $(C, D)$ and with a large rural and urban catchment areas $(B, E)$, all situated within England and Wales.

\begin{tabular}{|c|c|c|c|c|c|}
\hline & & $\begin{array}{l}\text { No. of } \\
\text { beds }\end{array}$ & $\begin{array}{l}\text { Days } \\
\text { Observed }\end{array}$ & $\begin{array}{l}\text { Hours } \\
\text { Observed }\end{array}$ & $\begin{array}{l}\text { In-situ } \\
\text { Interviews }\end{array}$ \\
\hline \multirow{2}{*}{ SITE A } & WARD & 28 & 19 & 110 & $\bigcirc 65$ \\
\hline & UNIT & 35 & 15 & 84 & 56 \\
\hline \multirow{2}{*}{ SITE B } & WARD & 29 & 16 & 75 & 58 \\
\hline & UNIT & 27 & 15 & 56 & 57 \\
\hline \multirow{2}{*}{ SITE C } & WARD & 30 & 15 & 79 & 56 \\
\hline & UNIT & 58 & 15 & 50 & 30 \\
\hline \multirow{2}{*}{ SITE D } & WARD & 24 & 15 & 67 & 16 \\
\hline & UNIT & 53 & 15 & 53 & 43 \\
\hline \multirow{2}{*}{ SITE E } & WARD & 28 & 15 & 78 & 19 \\
\hline & UNIT & 30 & 15 & 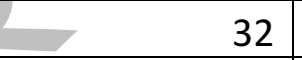 & 36 \\
\hline \multicolumn{3}{|c|}{ Totals } & 155 & 684 & 436 \\
\hline
\end{tabular}

Table A: Data collected from each site and ward.

Access to the sites was negotiated with hospital and ward managers in advance of planned field work. Patients within these settings with a diagnosis of dementia were identified through ward nursing handover notes, patient records and board data with the assistance of ward staff. Following the provision of written and verbal information about the study, and the expression of willingness to take part, written consent was taken from patients, staff and visitors directly observed or spoken to as part of the study.

Non- participant observations were conducted within 5 trauma and orthopaedic wards and 5 medical assessment units, areas of the hospital identified as having high levels of admission of people living with dementia. Across these sites, 155 periods of observational fieldwork was carried out between July 2015 and February 2017. Observations lasted between two and 12 hours of observation (median $=4$ hours), with the length of observation influenced by activity within the ward on that day. 
Observations focused on ward staff as they delivered care to patients living with dementia using 'thick description' to turn events into detailed descriptive accounts (Clifford, 1973.) Observations were taken by a researcher positioned in the corridor of the ward, with a view of a specific bay or bays, but without infringing on patient's dignity or personal space. Observations were written up during and immediately after the period of observation. Overall this produced approximately 600,000 words of fieldnotes.

Ethnographic interviews took place in situ on the ward during the delivery of everyday care, lasting from 1 minute to 10 minutes in length depending on the demands of the setting. Interviews were recorded as fieldnotes. In total 436 of these interviews took place with the ward population as staff cared for people living with dementia. This included not only nurses and health care assistants (nursing team members who provide direct patient care and who do not require formal training or registration with a professional body ) but also porters, hosts, specialist nurses, therapists, clinicians, registrars, and importantly people living with dementia, and their carers and families. This allowed the researchers to question what staff were doing and why, and what were the caring practices of ward staff when interacting with patients living with dementia.

Data collection (observations and interviews) and analysis were informed by the analytic tradition of grounded theory (Glaser \& Strauss 1967), which supported our focus on examining the phenomena or processes of resistance and refusal of care, rather than on the setting itself (Charmaz, 2014). There was no prior hypothesis testing and we utilized the constant comparative method and theoretical sampling whereby data collection (observation and interview data) and analysis are interrelated (Glaser \& Strauss 1967, Corbin \& Strauss 1990) and are carried out concurrently (Green 1998, Suddaby, 2006). The flexible nature of this approach is important, because it can allow us to increase the 'analytic incisiveness' (Charmaz and Mitchell, 2001:160) of the study. Preliminary analysis of data collected from individual sites informed the focus of later stages of sampling, data collection and analysis in other sites. 
To optimize the generalizability of our findings (Herriott and Firestone, 1983) our approach emphasizes the importance of comparisons across sites (Vogt, 2002), with theoretical saturation achieved following the search for negative cases, and on exploring a diverse and wide range of data. When no additional empirical data were found, we concluded that the analytic categories were saturated (Glaser and Strauss, 1967; Saunders et al, 2017).

Grounded theory and ethnography are complementary traditions, with grounded theory strengthening the ethnographic aims of achieving a theoretical interpretation of the data, whilst the ethnographic approach prevents a rigid application of grounded theory (Charmaz and Mitchell, 2001). Using an ethnographic approach can mean that everything within a setting is treated as data, which can lead to large volumes of unconnected data and a descriptive analysis (Atkinson and Coffey, 1996). A blended approach provides a middle ground in which the ethnographer, often seen as a passive observer of the social world, can use grounded theory to provide a systematic approach to data collection and analysis that can be used to develop theory to address the interpretive realities of participants within this setting (Charmaz and Mitchell, 2001).

Field notes of observations and near verbatim text were written up into word files (Emerson et al, 2011; Van Maanen, 2011). All sites, individuals, and data collected were anonymised and sorted in line with the Data Protection Act 1998, and NHS England Data Protection Policy 2014. Storage of the data is managed by the Cardiff University Information Security Framework Program. Observations on wards were undertaken by researchers with up to date Good Clinical Practice and Protection Of Vulnerable Adults (POVA) certification. Both Researchers hold PhDs and work within healthcare schools at UK Universities. They are not registered medical professionals, and held no regulatory duty of care at the time of the fieldwork. Fieldwork was guided by agreed procedures for researchers to ensure ethical practice in relation to responding to patient distress and / or the observation of substandard care. Ethical approval was granted by Wales REC3 (15/WA/1901). 


\section{Findings}

The findings of our study comprised of two overall themes, resistance and organisation. The theme of resistance examines the many forms by which resistance was identified and categorised within the ward, and the ways in which these manifestations had become an expected and accepted feature of everyday acute care routines. The theme of organisation identifies the organisational trap that staff and patients find themselves in within the ward, not only in lacking the support to prevent or counter resistance, but instead trapped in an organisational culture of bedside care that provokes and escalates resistance to care.

\subsection{Resistance: a routine part of everyday care}

Overall, the analysis revealed very high levels of resistance to care amongst people living with dementia within acute hospital wards. It was a common feature of ward life; every patient living with dementia we observed, resisted care to some extent or at some time during our period of observations. In total, we identified 1,052 (Trauma and Orthopaedic =523, Medical Assessment Units=529) incidents or episodes of resistance to care. This could manifest in a number of ways including physical resistance, verbal resistance, or refusal to comply with the timetabled rounds of the ward. Resistance was typically described by staff as a natural feature of a dementia diagnosis, and therefore a behaviour to be expected within and a feature of every shift, which they discussed as coming in waves, or being worse at particular times of day or within certain wards or bays.

\subsubsection{Physical resistance}

Physical resistance took many forms. While aggressive physical behaviours towards staff were observed, these actions were far outweighed by passive forms of physical resistance. This included pushing away trays or equipment, turning away from staff, or attempting to stand, walk, or leave the ward.

People attempting to get out of their bed or the bedside chair and, if that was achieved, to stand and walk (or as it was ubiquitously described, to 'wander'), was interpreted by ward staff as a key form of resistance. This was the most overt and 
commonly observed resistive activity and was consistently high across all wards. This response to care was expressed by the most immobile patients, such as a person who was only able to clutch at or pull and 'rattle' the raised rails at the sides of their bed containing them; people sitting in their chair who were able to push down on the arms of the chair in an attempt to stand up; to people who were able to walk away from the bedside. This behaviour was of immediate concern for staff if the person was able to raise themselves or stand, raising urgent concerns around the risk of falling or a patient leaving the ward.

In one example, typical of a standard afternoon of interactions between staff and patients (Site E, Trauma and Orthopaedic, day 11), an 86yr old woman living with vascular dementia had been admitted following a 'fall' and over one afternoon made multiple attempts to get out of bed. Every time she tried to leave the chair or the bed the health care assistant in the bay immediately responded and repeatedly helped her to sit back in the chair ('let's sit you up for lunch') or lead her back into bed. Ward staff (particularly the health care assistants) encouraged her to sleep, arranging the bed and holding her hand to try to calm her. However, every time the patient appeared settled, she would immediately return to reach for something, to sit up, or get out of bed and stand. Her need to get up was related to the anxieties she expressed about her home, where her house keys were and who was picking her up and taking her home. Throughout this afternoon, staff responses to her started with distraction (lunch), to repeatedly questioning her 'what are you doing?' , 'where do you want to go?', which escalated to giving her clear directives 'you need to rest' and enrolling the requirements of the wider institution by suggesting other people had the power to decide 'We need to talk to the doctors first' and 'your sons will sort it out for you'. As ward staff talked to her, they also continued to subtly restrict her movement throughout the shift. They start with tucking the blanket over her legs and placing trolley in front of her in the chair, and within the bed use pillows around her body, cover her up with the sheets and sit next to her holding her hand. Physical resistance was subject to a variety of responses from staff, dependent on their perceptions of risk to the patient. Patients lacking the strength or mobility to achieve their aim of getting out of bed, standing or walking, were typically assessed 
by staff as not being an immediate concern. Here, a 94-year-old man with a fractured hip and pneumonia, continued to rattle and pull at the raised bars at the sides of his bed. However, even though he did this forcefully and over an extended period during the shift, an indication that he appears to be distressed, the team did not intervene and continued their focus on the ward routines around him:

The health care assistant returns to the bay with the mobile blood pressure unit from taking the measurements from patients in the cubicles. The nurse finds the yellow charts for him have returned and are open on the trolley at the end of the bed and updates them. He is a very thin man wearing a hospital gown lying in bed - the side rails of the bed are up and he is holding on tight to the left side bar and shaking it and rattling it as if trying to get out. He moans and sounds very distressed. The nurse continues and moves on with the medication round to other patients in the bay and as she is doing this, he holds tightly onto the raised side bar on his bed, continuing to rattle and pull at it. [Site A, Trauma and Orthopaedic ward, day 4]

Physical resistance was seen by staff as less problematic when the person was believed to be contained safely within a bed, as in the example above. It would often only be prioritised if it placed the patient at risk of falling. Similarly, walking or walking unaccompanied within the ward was almost always discouraged and problematised as a form of resistance that typically extended to older patients within the ward.

\subsubsection{Verbal resistance}

Shouting often became an accepted background noise on wards. Once it was established the patient was not at risk, ward staff seemed able to ignore shouting, allowing them to continue with other tasks and patients. Often, however, this would lead to resistance by contagion, where the shouting of one patient triggered resistance, both physical and verbal, in others. The case below of two patients, both women in their 80 s admitted with frailty is a typical example of this: 
The lady from bay 4 yesterday has been moved to bay 2. Lying flat on her back on bed 6, she is again loudly shouting 'Take me home, I want to go home', which the lady across the bay in bed 2 has begun to repeat. The first lady begins to respond to this 'it's shameful, shocking', then continues to call out, left to talk to herself, while the lady in bed 2 is calmed by a visitor at her bedside. A new patient is brought to bed 1, asleep on the bed as it is wheeled into place. Pointing to this bed the lady in bed 6 shouts 'Back home!.. don't put her there, I don't want that'. When either lady shouts, it prompts the other, who begins to shout again as well. The two patients are seemingly triggering one another but neither being responded to. [Site 5, Medical Assessment Unit, Day 15]

Staff usually paid little attention to such perceived low risk behaviours, but these established patterns of resistance then became recognised as problematic in relation to the potential for escalation and obstructing the delivery of timetabled care.

\section{3..1.3 Resistance towards the timetabled rounds of the ward}

A common way in which ward staff identified resistance to care, was when patients failed to submit to the established timetables of bedside care within the ward, which includes the everyday rounds of mealtimes, medications, personal care and observations at the bedside.

\subsubsection{Mealtimes}

Mealtimes were a critical trigger for resistance during the timetabled ward care and it was the routine where resistance was most visible. Often this would be in the form of a clear sign that a person living with dementia did not want to eat (verbal or physical) or the absence of communication (silence in response to often repeated requests). This was almost always viewed by staff as resistive and a feature of the person's dementia diagnosis that had to be overcome. Ward staff typically prioritised speed, efficiency, and consumption, which meant that mealtimes could become a battle of wills between patient and staff. The impact of repeated attempts to encourage people to eat and the often invasive approaches staff used to try to 'feed' someone was that it typically made people angry, increasing their anxiety and distrust of staff, as demonstrated in the example below. 
Health care assistant takes over a meal of pureed sausage, pureed peas, mash and gravy: 'I have a lovely lunch here for you, do you want to try it?' She is sitting next to the patient and puts a clean linen pillowcase across her chest and gives her a large metal spoon, 'you try it, hold the spoon'. She helps her told the spoon and also holds it and guides it into the mash and then guides it towards her mouth. But this woman pushes her hand away from her mouth and puts the spoon back onto the plate. The health care assistant then once again uses the spoon to scoop quite a large amount of mash and gravy onto the spoon and guides it back to her mouth and she takes a tiny bit. The health care assistant is very encouraging and moves on to the dessert, 'I have apple and custard'. She sits next to her and puts a bit on a large metal spoon, 'Let's try again', she tries to put the spoon into her hand. In response, the woman takes it and pushes it away extremely forcefully, it is so fast and unexpected that the health care assistant jumps out of the chair in shock. She turns to the woman: 'let's stop there'. However, this patient now looks very anxious and wide eyed as she sits in the chair [Site E, Trauma and Orthopaedic, Day 5]

Even when people clearly indicated that they did not want to eat, ward staff typically would often continue to spoon feed the person and make at least one further attempt before stopping.

\subsubsection{Medication}

The medication round was always a time of increased urgency and anxiety for nursing staff, driven by perceived constraints within the ward timetable and the importance of patients taking all of their (typically multiple) medication. Staff expressed a clear sense of relief and accomplishment if the round was completed without significant perceived resistance. Common reasons for people resisting included changes to their medication (including variation from their regular brand, dose, shape and colour) or apprehension of the side effects from new medication. While a typical feature of the round, this was always viewed by staff as problematic and resistive, increasing their anxiety and frustration:

The nurse goes over to the patient with a pill pot. She has a loud voice and is very strident, 'I have one little tablet from the doctors who saw you today'. 
She puts it into her palm and the woman looks at it closely: 'It's not the blue one'. She picks it up and puts it in her tea cup straight away. The nurse is clearly extremely exasperated: 'Can I explain to you! Because you have been seen by the psychiatrist today he has given you this'. Patient: 'No, it's rubbish'. She is very clear that she doesn't want it. Nurse: 'He has prescribed it, Ok, you don't want to take it'. She is very exasperated and puts the rest of the medication into her personal drug cabinet, saying to herself and the wider ward: 'It's the first one that has been prescribed!' She sounds very frustrated and writes in her bedside notes and says to me: 'They will say you haven't tried!' [Site E Trauma and Orthopaedic, Day 8]

\subsubsection{Personal care}

Ward staff always explicitly sought the person's permission to carry out personal and intimate care. However, they also typically started work on their body immediately, whether the patient consented or otherwise, and continued with the task at hand, highlighting the perceived essential nature of this care. In this context, the person could have just woken up, have difficulties communicating verbally, or appear anxious or afraid of moving. Any response patients had to this type of request would frequently take the form of a physical or verbal resistance, while staff typically continued their focus on completing care. For staff, this work was of additional importance to the wider ward of presenting a neat and tidy patient, bedside, and bay, to meet the timetable and routines of the ward, and expectations of the institution. As with the other types of resistive behaviour detailed earlier, these behaviours were interpreted by staff as a feature of the dementia diagnosis and interpreted as the person lacking capacity, forming behaviours that must be challenged and overcome.

\subsection{The organisational trap: locked into cycles of care and the consequences}

It was identified that the timetabled routines of bedside care had the impact of increasing the focus on and value of some aspects of ward work over others. 
Institutional timetables, and the high value set on achieving efficiency and reducing perceived risks to patients, dictated the priorities set by staff, ensuring a focus on the delivery of essential everyday planned care over patient need or mood in that moment. Staff felt constrained into delivering routines of care that triggered cycles of resistance and escalation.

\subsubsection{Cycles and escalation of resistance}

The majority of encounters with people living with dementia occurred during the timetabled routines of the ward. The majority of these encounters were unproblematic. However, when examined across whole bays, wards, or units, resistance was a common occurrence and it was rare for a timetabled round of bedside care within any bay of 4-8 people to be completed without some form of resistance. Routine cycles of conflict and resistance during timetabled care were observed to be repeated every shift, often with different staff, rehearsing and duplicating the interactional performance and routines to complete timetabled care with the same patient. Resistance would often be contagious, with one person's resistance agitating another, leading to escalating cycles within a bay or ward area. When ward routines and timetables were interrupted by a number of people resisting care, staff responses typically focused on short term management and containment so that they could focus on completing the task in hand and the timetables of the ward, rather than responding to individual anxieties and fears. However, if these subtle signs were not acknowledged, then they typically escalated into a longer-term entrenched pattern of resistance.

\subsubsection{Routines of the ward and directing care.}

As illustrated earlier, the routine of the ward took priority over the needs of the individual patient living with dementia. These timetabled routines were often determined outside of the nursing team, by organisationally mandated care standards or determined by the schedules of other work teams, for instance, the ward rounds of the medical teams or the auxiliaries who delivered and cleared away meals. The routines were typically shared across both Trauma and Orthopaedic 
wards and Medical Assessment Units at each site, despite the very different needs of patients within each. This left ward staff with very little flexibility to respond to patient needs that fell outside of scheduled and quantifiable work. In addition, the organisation of bedside work into a series of tasks, enabled the timetable to be delivered, but meant for individual staff that their interactions with individual patients were very limited. For example, a nurse leading a medications round might meet every patient on the ward twice during a shift but at no point, have the opportunity to learn how best support that individual or develop a relationship with them.

Typically, as in this example below, the ward teams' approach to patient care focused on carrying out specific care for the person at the bedside with the goal of completing the wider ward routines and timetable. This extended to their response to any perceived resistance, which was typically to view these as potential interruptions to the work of the ward and to continue and try to complete timetabled care. If a patient responded by resisting, or by saying 'no' to that care or intervention, although staff would acknowledge this in their talk at the bedside, they typically did not address these concerns. Instead they reminded the patient of the expectations of the ward and typically focussed on the completion of the immediate task. However, this approach appeared to exacerbate resistance and ward staff acknowledged the need for strategies, such as in this exchange with a group of nurses and health care assistants working together on a shift.

'You have to make decisions, which is the most important'

'They are all different, fighting with us, this has an impact on all the patients stress...we do our best....there are stressful moments'

'I know what's going to happen, you start to understand what reactions are going to be, so you adapt your way of being- it's hard work!'

'You know what to do' [Site E, Trauma and Orthopaedic, Day 4] Ward staff expressed a clear sense of relief and accomplishment if these rounds were completed without perceived resistance and delay. Such relief would be short-lived, prefacing as it did the next round of timetabled work. Throughout the shifts, the 
timetables of institutionally mandated routines influenced how work was organised and scheduled, with priority given to certain aspects over others. This prioritisation of some work over others, in turn, led to patterns of resistance.

Patients living with dementia typically were physically slower, needed longer, did not appear to respond immediately, or resist care. However, any perceived delays or challenges to the timetables often caused staff high levels of stress and anxiety. When the timetables of the ward started to break down, this would lead to increasing noise of patient's personal buzzers (these could be particularly loud and piercing in some wards) and cries for help, which could increase the intensity of the ward. As one health care assistant tells us 'the buzzers haunt me, I hear them in my dreams' [Site A, Trauma and Orthopaedic, day 10]. Staff were then faced with competing needs of the routine timetable or the urgency of individual people living with dementia.

In the example below, a woman with a diagnosis of dementia has been repeatedly trying to get out of bed (and shouting at staff) for approximately four hours. She is very slight and frail, and looks unable to support herself. She repeatedly uses the raised side bars of the bed to pull herself up and raise her feet off the bed. The ward is understaffed as a young man with a psychiatric admission has left the ward and staff are attempting to find him.

The lady in bed 6 still has a health care assistant next to her and is still trying to stand up. RN has observed but is not intervening. The health care assistant is getting sterner, making her sit back on bed, not asking her to sit. Health care assistant is clearly concerned but is getting increasingly annoyed/stern with her now. The health care assistant has asked for help from another health care assistant passing the bay. They curtain the patient, who can be hear loudly shouting 'GET OFF ME NOW' 'NO!' 'get off my hands'. Health care assistant is asking her, by her first name, to hold her hand, trying to reassure her. The curtain is drawn back and the patient seems to be very tightly tucked into her bed with a blanket. She is now lying back under blanket, looking around and tapping her feet, no longer trying (or able?) to stand up. 
The surrounding beds are calm, most with visitors. After a brief respite the patient begins raise herself up again, health care assistant again reprimands her to lie back down verbally, very firmly, which she does. The health care assistant comes over to nurses' station and apologises for her 'little nervous breakdown' earlier. While dealing with the patient she got very upset and, following their interaction, went to the sluice room, closed door after, and was quite clearly crying, visibly upset by having to restrain and argue with her patient. [Site A, Medical Assessment Unit, Day 1]

As in this example, the results were to manage people living with dementia on the ward, typically through containment at the bedside and restraint. The emotional toll resultant from these patterns and this perceived lack of support was often clear on both patients and ward staff.

\subsubsection{Expectations of high dependency and resistance}

People living with dementia, were often very capable of many types of self-care during their admission (eating meals, walking independently, being continent). However, this was typically independence that was undermined by the type of care on offer, denied by the organisation of timetabled delivery of care within the wards and this had a further impact on shaping ward staff understandings of dementia. Staff appeared to expect that people living with dementia would need support at mealtimes, be unable to walk independently, and be at high risk of falling, with often presumed incontinence. This led to routine practices of care that increased staff workloads and also limited opportunities for people living with dementia to rehabilitate and increase their independence.

For example, it was an everyday assumption that most people living with dementia could not eat meals without assistance. People were often identified by staff as a 'feeder' (i.e. that they needed spoon feeding), even if they demonstrated during other shifts that they could eat independently or with minimal support. This classification typically appeared to be made by the ward team on the basis of a dementia diagnosis and it was rare for individuals to be asked by ward staff if they 
wanted support with the meal, to support them in eating independently or to see if this was possible. However, these assumptions about who can and cannot eat independently were features of the timetabled delivery of care and could trigger resistance to care:

The one-to-one health care assistant goes over to the nurse - I am going to feed (a woman in her 80s who has a diagnosis of dementia. She been lying in bed and has been pulling off her sheets, stretching out her arms to staff as they pass by and calling for 'mummy') The health care assistant sits in the chair next to her and is very efficient and functional, shovelling large spoons of soup and then the sponge pudding. She leaves the sandwiches.

'NAUGHTY!' the one-to-one health care assistant exclaims 'you are getting it all over you!' A bit of soup has been spilled, but these are large mouthfuls that the one-to-one health care assistant is shovelling into her mouth quickly one after another - 'open up'. At one stage she tries to stop the one-to-one health care assistant and taps her on the arm. She hardly has any strength, but the one-to-one health care assistant says loudly 'WHY ARE YOU HITTING ME?', and continues to shovel large spoons of sponge pudding and custard into her mouth. [Site D, Trauma and Orthopaedic, day 11]

This classification and the approach to assessing people's ability to eat independently had a wider impact on how individuals with dementia were viewed and classified by staff, influencing their wider understandings of the capacity, autonomy, independence and the ability of people living with dementia to make decisions, and in turn impacting on their care pathways.

\subsubsection{Routines exclude interpersonal care and communication}

Staff often discussed their lack of continuity and 'not knowing' the patients within their assigned bay, which meant that they were not able to establish patterns of behaviour or of ways of working with individuals. There was always an expectation that other specialist wards were able to support this way of working.

Importantly, nurses were never observed feeling able to sit with, spend time with, or listen to patients. Talking to patients only occurred as staff worked with them, to complete timetabled care. However, even during this routine care work, this talk 
was limited. It typically addressed the person by locating them very clearly in relation to the reality of where they were, what had happened to them and what was going on around them. The focus was on reorienting and locating the person to the expectations of the ward and to manage the person within the routines of the ward. Importantly, this talk was typically rhetorical and did not require or expect the person to respond or assent. In response to any perceived resistance, this talk focussed on reminding people of the expectations of the institution, emphasising that there was no choice for either the person or the ward team caring for them.

\section{Discussion}

This study explores the ways in which resistance to care by people living with dementia manifests within the acute setting and is understood, classified and subsequently managed by ward staff. Earlier in the paper we cited claims that the hospital social environment and its relationship to patient resistance is underexamined and that the evidence base lacks research on the extent of resistance to care, how it manifests, or how it should be managed, within the acute hospital setting (Cheong et al, 2016; Dewing \& Dijk, 2014; Keady \& Jones, 2010; Pizzacalla et al, 2015; Rantala et al, 2014; Werner et al, 2002). The findings set out here are an important step in addressing these gaps.

This study found that resistance was a routine and expected part of everyday care on the Medical Assessment Units and Trauma and Orthopaedic wards that were studied, occurring frequently and manifesting physically and verbally. The timetabled rounds of the ward (mealtimes, medication rounds, planned personal care) significantly shaped patient and staff experiences and behaviours. Staff perceptions that resistive behaviours could be explained by and were a feature of a dementia diagnosis, and the perceived primacy of completing timetabled care for staff, resulted in a failure to respond positively to individual patient needs in the moment. These routinised ward cultures typically triggered further cycles and escalation of patient resistance to bedside care.

The ethnographic approach allowed an examination of a poorly documented, and typically undocumented, phenomena within acute wards and to identify the sheer 
scale of resistance to care by people living with dementia within the acute setting. Importantly, the underlying triggers for a person's resistance were explored, and how this relates to aspects of the organisation and delivery of bedside care during an admission. Importantly, this highlights that resistance typically did not take the form of isolated events where only one or a small number of individuals within the ward or bay resisted care. Every person we observed was identified by ward staff as resisting care at some point.

In isolation, each of these instances of resistance and refusal appeared relatively minor in terms of its overall impact on the ward, if not the patient, and often easily rectified. In combination, however, these minor acts of resistance, carried out by many patients, if not simultaneously, then in close association, had a significant influence on ward cultures, and approaches to and recognition of people living with dementia within the wards. In keeping with our approach, we did look for deviant cases during the course of the fieldwork. However, these patterns were consistent across wards and institutions.

These findings illuminate an area of nursing practice that has been under-researched in this setting. In particular, the impact of the social environment on the manifestation of resistive behaviours were identified, and have thus shone an important light on patient and staff experiences in this setting. Other research has identified the importance to older people in acute settings of the relational aspects of care, especially people living with dementia, and our findings illuminate how, in practice, the importance of relational care can be overshadowed by other drivers.(Bridges et al. 2010).

Through its detailed observations, this study has established the importance of good relational care to patient experiences, but also to patient outcomes. Hospital patients with cognitive impairment have higher length of stay, are more likely to be admitted to a care home on discharge (having come from their own home) and at higher risk of dying in hospital than patients who are not cognitively impaired (Zekry et al. 2009, Mukadan \& Sampson, 2011, Fogg et al., 2017, 2018). Some of these poorer outcomes may be attributable to poorer underlying health linked to the cognitive impairment itself, for instance, cognitive impairment means a higher chance of being 
admitted to hospital in a malnourished state (Mukadan \& Sampson, 2011, Fogg et al., 2017). However, research to date suggests that the care in hospital is a major underlying factor to these risks (Alzheimer's Society, 2009, Mukadan \& Sampson, 2011, Fogg et al. 2018). This study's findings may explain, at least in part, what is happening here. If the way that care is delivered leads people to resist essential bedside care, the medications, food and drink that prevent their health deteriorating, and if the type of care means that they are prevented from staying as mobile and independent as possible, it is unsurprising that people living with dementia have poor outcomes and experiences.

The findings have also shown the strong influences from the wider institution on the ways in which ward staff are able to act and the organisational constrains in the type of care they are able to give. Other research suggests that moral distress and burnout follow if nurses experience organisational barriers that limit care quality (Nordam et al. 2005, Edberg et al. 2008, Bridges et al. 2012), and our research deepens understanding of the challenges that nursing staff can face in their everyday work. Our findings reflect that frontline staff did little to mediate between institutional drivers and the needs of the patients in front of them. However, the high levels of staff anxiety and upset we observed suggest a moral distress associated with the deprioritisation of actual patient need in the moment. Our findings indicate that staff felt blocked from delivering the care they saw was needed and did not feel in control of how care priorities were determined. We found little evidence of more senior members of the institution interpreting or mediating these organisational drivers for frontline staff. We know from other research that nurses do not often control the conditions in which they work due to a curbing of professional autonomy in publicly funded health care, and the particular position that nursing holds in the professional and managerial hierarchy (Bridges at al. 2017; Davies 1995; Exworthy \& Halford 1999; Hyde et al. 2016). Again, where this study contributes is by demonstrating how this positioning of nursing as a profession within the acute setting influences the ways in which they can and cannot act in relation to patient need. 
Staff training in person-centred dementia care is often hailed as a solution to the widely recognized problems in the provision of care (Clissett et al 2013, Houghton et al 2016, Tay et al 2018). The findings in this study suggest that the problem is more of a cultural and organisational issue than a training issue, although training would certainly help to address the issues we observed. This work looked at two kinds of in-patient specialty and it would be interesting to explore the transferability of these findings to other acute settings where staff may hold greater expertise in caring for people with dementia, for instance, on medical wards for older people, or specialist dementia wards. The institutional timetable would presumably still exist but it may be that higher expertise, a more person-centred team culture and strong clinical leadership could yield different results. For these reasons, we limit our claims for transferability to general acute care ward settings with similar patient populations and staff profile.

The data are limited in understanding staff perceptions of how change could improve patient experiences and reduce resistive behaviours, and it may be that a formal interview study would reveal further insight. Overall, an in-depth ethnographic approach across a number of hospital settings has yielded important insights into how resistive behaviours are triggered and manifest in acute care settings, how they are understood, classified and managed by ward nursing staff, and the consequences for people living with dementia.

\section{Conclusion}

Person-centred approaches towards the care of people living with dementia have long been accepted as best practice. Our findings illustrate that nursing staff struggle to deliver person-centred dementia care in acute care settings where the institutional drivers of routines, efficiency and perceived risk management and reduction do not appear to be mediated by frontline staff or clinical leadership. Cycles of resistance in response to organisationally mandated timetables of care can result in poor care experiences for patients, and emotional and physical burnout for staff. More research is urgently needed to understand how institutional goals and individualised needs of people living with dementia can be better aligned. 


\section{References}

Alzheimer's Society, 2009. Counting the cost: caring for people with dementia on hospital wards. Alzheimer's Society, London.

Atkinson, P., Housley, W., 2003. Interactionism, BSA New Horizons in Sociology. SAGE Publications.

Bridges, J., Flatley, M., Meyer, J., 2010. Older people's and relatives' experiences in acute care settings: systematic review and synthesis of qualitative studies. Int J Nurs Stud 47. https://doi.org/10.1016/j.ijnurstu.2009.09.009

Bridges, J., May, C., Fuller, A., Griffiths, P., Wigley, W., Gould, L., Barker, H., Libberton, P., 2017. Optimising impact and sustainability: a qualitative process evaluation of a complex intervention targeted at compassionate care. BMJ Qual Saf bmjqs-2017.

Bridges, J., Nicholson, C., Maben, J., Pope, C., Flatley, M., Wilkinson, C., Meyer, J., Tziggili, M., 2013. Capacity for care: meta-ethnography of acute care nurses' experiences of the nurse-patient relationship. J. Adv. Nurs. 69, 760-772.

Charmaz, K., 2014. Constructing grounded theory. Sage

Cheong, C.Y., Tan, J.A.Q., Foong, Y.-L., Koh, H.M., Chen, D.Z.Y., Tan, J.J.C., Ng, C.J., Yap, P., 2016. Creative Music Therapy in an Acute Care Setting for Older Patients with Delirium and Dementia. Dement. Geriatr. Cogn. Dis. Extra 6, 268-275. https://doi.org/10.1159/000445883

Clissett, P., Porock, D., Harwood, R.H., Gladman, J.R.F., 2013. The challenges of achieving person-centred care in acute hospitals: A qualitative study of people with dementia and their families. Int. J. Nurs. Stud. 50, 1495-1503.

Davies, C., 1995. Competence versus Care? Gender and Caring Work Revisited. Acta Sociol. $38,17-31$.

Dewing, J., Dijk, S., 2014. What is the current state of care for older people with dementia in general hospitals? A literature review. Dementia 15, 106-124.

Edberg, A.-K., Bird, M., Richards, D.A., Woods, R., Keeley, P., Davis-Quarrell, V., 2008. Strain in nursing care of people with dementia: nurses' experience in Australia, Sweden and United Kingdom. Aging and Mental Health 12 (2), 236-243.

Emerson, R.M., Fretz, R.I., Shaw, L.L., 2011. Writing ethnographic fieldnotes. University of Chicago Press.

Exworthy, M., Halford, S., 1999. Professionals and the New Managerialism in the Public Sector. Open University Press.

Featherstone, K., Northcott, A., Harden, J., Tope, R., Bale, S., Harrison-Dening, K., Bridges, J. (In Press) Resistance to care by people living with dementia in acute hospital wards: an ethnographic study. Health Services and Delivery Research

Fogg, C., Griffiths, P., Meredith, P., Bridges, J., 2018. Hospital outcomes of older people with cognitive impairment: An integrative review. Int. J. Geriatr. Psychiatry.

Fogg, C., Meredith, P., Bridges, J., Gould, G.P., Griffiths, P., 2017. The relationship between cognitive impairment, mortality and discharge characteristics in a large cohort of older 
adults with unscheduled admissions to an acute hospital: a retrospective observational study. Age Ageing 46, 794-801.

Geertz, C., 1973. The interpretation of cultures: selected essays. New York: Basic Books

Glaser, B., Strauss, A., 1967. The discovery ofgrounded theory. London: Weidenfeld and Nicholson 24, 288-304.

Green, J., 1998. Commentary: grounded theory and the constant comparative method. Br. Med. J. 316, 1064-1066.

Greenhalgh, T., Swinglehurst, D., 2011. Studying technology use as social practice: the untapped potential of ethnography. BMC medicine, 9(1), 45.

Houghton, C., Murphy, K., Brooker, D., Casey, D., 2016. Healthcare staffs' experiences and perceptions of caring for people with dementia in the acute setting: Qualitative evidence synthesis. Int. J. Nurs. Stud. 61, 104-116. https://doi.org/10.1016/j.ijnurstu.2016.06.001

Hyde, P., Granter, E., Hassard, J., McCann, L., 2016. Deconstructing the Welfare State: Managing Healthcare in the Age of Reform. Taylor \& Francis.

Ishii, S., Streim, J.E., Saliba, D., 2012. A conceptual framework for rejection of care behaviors: Review of literature and analysis of role of dementia severity. J. Am. Med. Dir. Assoc. 13, 11-23. https://doi.org/10.1016/j.jamda.2010.11.004

Kable, A., Guest, M., McLeod, M., 2011. Organizational risk management of resistance to care episodes in health facilities. J. Adv. Nurs. 68, 1933-1943. https://doi.org/10.1111/j.1365-2648.2011.05874.x

Keady, J., Jones, L., 2010. Investigating the causes of behaviours that challenge in people with dementia. Nurs. Older People 22, 25-29. https://doi.org/10.7748/nop2010.11.22.9.25.c8061

Moyle, W., Olorenshaw, R., Wallis, M., Borbasi, S., 2008. Best practice for the management of older people with dementia in the acute care setting: a review of the literature. Int. J. Older People Nurs. 3, 121-130.

Mukadam, N., Sampson, E.L., 2011. A systematic review of the prevalence, associations and outcomes of dementia in older general hospital inpatients. International Psychogeriatrics $23(3), 344-355$.

Nordam, A., Torjuul, K., Sørlie, V., 2005. Ethical challenges in the care of older people and risk of being burned out among male nurses. Journal of Clinical Nursing 14 (10), 12481256.

Pizzacalla, A., Montemuro, M., Coker, E., Martin, L.S., Gillies, L., Robinson, K., Pepper, H., Benner, J., Gusciora, J., 2015. Gentle Persuasive Approaches: Introducing an Educational Program on an Orthopaedic Unit for Staff Caring for Patients with Dementia and Delirium. Orthop. Nurs. 34, 101-107.

Quinlan, E., 2009. The 'actualities' of knowledge work: an institutional ethnography of multi-disciplinary primary health careteams. Sociology of health \& illness, 31(5):625641 
Rantala, M., Kankkunen, P., Kvist, T., Hartikainen, S., 2014. Barriers to postoperative pain management in hip fracture patients with dementia as evaluated by nursing staff. Pain Manag. Nurs. 15, 208-219. https://doi.org/10.1016/j.pmn.2012.08.007

Sangars, D., Taylor, M., Sangars, B., 2014. Inside the DoLS house, A Freedom of Information Act survey of variations in the use of Deprivation of Liberty Safeguards in England. Age Ageing 43, ii10-ii10.

Saunders, B et al. "Saturation in qualitative research: exploring its conceptualization and operationalization" Quality \& quantity vol. 52,4 (2017): 1893-1907

Suddaby, R., 2006. From the editors: What grounded theory is not. Academy of management Journal, 49(4), 633-642.

Tay, F.H.E., Thompson, C.L., Nieh, C.M., Nieh, C.C., Koh, H.M., Tan, J.J.C., Yap, P.L.K., 2018. Person-centered care for older people with dementia in the acute hospital.

Alzheimer's Dement. Transl. Res. Clin. Interv. 4, 19-27. https://doi.org/10.1016/j.trci.2017.11.003

Van Maanen, J., 2011. Tales of the field: On writing ethnography. University of Chicago Press.

Zekry, D., Herrmann, F.R., Grandjean, R., Vitale, A.M., De Pinho, M.F., Michel, J.P., Gold, G., Krause, K.H., 2009. Does dementia predict adverse hospitalization outcomes? A prospective study in aged inpatients. International Journal of Geriatric Psychiatry: A journal of the psychiatry of late life and allied sciences 24 (3), 283-291. 